\title{
Kritische Stadtforschungen
}

Ein Gespräch über Geschichte und Produktionsbedingungen, Disziplinen

und Interdisziplinarität

Nina Gribat, Stefan Hoehne,

Boris Michel und Nina Schuster

Als Redaktion von s u b $\backslash \mathbf{u} \mathbf{r} \mathbf{b}$ a $\mathbf{n}$ begreifen wir uns als interdisziplinäre Redaktion, die eine interdisziplinäre Zeitschrift für kritische Stadtforschung macht. Zu unseren Gründungszeiten haben wir viel über den Begriff der Interdisziplinarität diskutiert und uns gefragt, ob wir nicht eher trans- oder postdisziplinär sind bzw. sein wollen. In unserer Redaktionsarbeit sind uns Fragen von Disziplinen dann überraschend oft begegnet und das war nicht immer einfach, zum Beispiel im Begutachtungsverfahren. Ein Zitat von Lefebvre (welcher Disziplin er auch immer angehörte) fasst dieses Spannungsfeld zusammen. In Die Revolution der Städte schreibt er 1970, dass die Komplexität des Urbanen bzw. des Städtischen „die Zusammenarbeit der einzelnen Disziplinen unerläßlich [macht]. Das Phänomen Verstädterung kann in seinem gesamten Umfang nicht von einer Spezialwissenschaft bewältigt werden. [...] Wenn man das zugibt bzw. postuliert, setzen die Schwierigkeiten erst ein. Wer kennt nicht die Enttäuschungen und Rückschläge, die man bei den sogenannten ,interdisziplinären' oder ,pluridisziplinären' Konferenzen erlebt. [...] Bald ein Dialog von Tauben, bald eine Pseudo-Begegnung ohne gemeinsame Standpunkte.“

Aufgrund dieser Überlegungen zur konstitutionellen Interdisziplinarität der Stadtforschung und gleichzeitig zu den Schwierigkeiten ihrer Umsetzung haben wir die Ressourcen unserer interdisziplinären Redaktion mobilisiert, um eine Debatte über kritische Stadtforschung und Interdisziplinarität zu starten, die wir auch in Zukunft gerne fortsetzen möchten. Erster Schritt dieser Diskussion war die Rekonstruktion einer Geschichte der Stadtforschung im deutschsprachigen Raum. Durch die Perspektive unterschiedlicher Disziplinen haben wir versucht, die Entwicklung der Stadtforschung nachzuvollziehen und dabei die Entstehung einer explizit ,kritischen` Besinnung und deren Veränderung in einen breiteren historischen Kontext einzubetten. Anhand dieser ersten Ergebnisse über die Geschichte der Stadtforschung skizzieren wir Züge einer Diagnose der heutigen Produktionsbedingungen kritischer Stadtforschung im deutschsprachigen Raum und formulieren Wünsche für ihre weitere Entwicklung. Am Gespräch beteiligt haben sich Vertreter_innen aus Geographie (Boris Michel), Architektur/Städtebau (Nina Gribat), Kulturgeschichte (Stefan Höhne) und Soziologie (Nina Schuster). 
s u b $\backslash \mathbf{u} \mathbf{r} \mathbf{b}$ a n (s $\backslash \mathbf{u})$ : Eine sozial-, kultur- und geisteswissenschaftliche Auseinandersetzung mit Städten setzte nicht nur in einer Zeit ein, in der die rapide wachsenden Städte in Europa zu einem gesellschaftlichen Problem wurden. Sie entwickelte sich auch zu einer Zeit, in der sich das, was heute an akademischen Disziplinen zugegen ist, herauszubilden begann. Wie haben also vor dem Hintergrund, dass die Grenzen der Disziplinen noch gar nicht so klar waren, die verschiedenen,Disziplinen'Stadt oder Urbanität als Thema und Forschungsgegenstand entdeckt?

Nina Schuster (NiS): Die Entstehung der Stadtsoziologie wird in die Zeit der zweiten industriellen Revolution datiert und eng mit der Entstehung und dem Anwachsen großer Städte verbunden. Für den Frühkapitalismus des 19. Jahrhunderts beschreiben Häußermann und Siebel zwei entgegengesetzte soziologische Reaktionen auf das Anwachsen der Städte und das Entstehen einer neuen sozialen Schicht: Einerseits sehen sie die dominante, konservative, manchmal reaktionäre Stadtkritik, die die industrielle Großstadt für das Elend in den Städten verantwortlich macht. Sie forderte die Abschaffung der Großstädte und die Rückkehr der Menschen in kleinere Städte und Dörfer. Andererseits existierte bereits die,progressive`Stadttheorie, die mit Friedrich Engels' Die Lage der arbeitenden Klasse in England (1845) beginnt. Sie sah in den neuen Verhältnissen eine Chance für grundlegende gesellschaftliche Umwälzungen und ging davon aus, dass die gesellschaftliche Dynamik sich bis zur Krise steigern würde, aus der schließlich eine Revolution wieder herausführen würde. Einige Jahre später entsteht mit Ferdinand Tönnies eine Soziologie, die diesen Fokus auf die Erfahrungen mit dem Leben in Großstädten weiter stärkt: Individualisierung und die Anonymität und Flüchtigkeit der Begegnungen sind für Tönnies' Gegenüberstellung von Gemeinschaft und Gesellschaft typisch großstädtisch und damit charakteristisch für die Gesellschaft, die im Gegensatz zur traditionellen Gemeinschaft von Arbeitsteilung und unverbindlichen Tauschbeziehungen geprägt ist. Diese Perspektive auf die moderne Großstadt baut Georg Simmel Anfang des 20. Jahrhunderts aus, indem er konträr zur dominanten Großstadtkritik Individualisierung und Anonymität in der Großstadt mit den Möglichkeiten der Stadt als Ort der Emanzipation von traditionellen Lebensformen assoziiert. Demgegenüber bemüht sich Max Weber in seiner historischen Rekonstruktion von Stadttypologien 1921 in Wirtschaft und Gesellschaft, sich einer Wertung des Phänomens Großstadt zu enthalten. Er bezieht sich besonders auf die ökonomischen, untergeordnet auch auf die politischen und sozialen, Aspekte der Herausbildung der verschiedenen Typen okzidentaler Städte bis zur Industrialisierung. Seine Darstellung hält sich mit Einschätzungen zu zeitgenössischen Stadtentwicklungen zurück.

Nina Gribat (NG): Das ist spannend, ich sehe in dieser sehr unterschiedlichen Bewertung der Großstadt in der Industrialisierung eine Parallele zur Architektur und zum Städtebau, vor allem im Übergang zur Moderne oder zum Funktionalismus. Grundsätzlich lässt sich an diese durch die Praxis des Planens und Bauens geprägte Disziplinen natürlich die Frage stellen, inwieweit deren Beschäftigung mit der Stadt oder mit Urbanität auch für Sozialund Geisteswissenschaftler_innen als ,Forschung' gilt - inwieweit also das 
Planen und Bauen auch einen eigenständigen Beitrag zum Erkenntnisgewinn darstellt. Ich möchte in meinen Redebeiträgen hier einfach mal davon ausgehen (ohne jedoch näher bestimmen zu wollen, wie dieser Beitrag genau aussieht). Ich behandle also Entwürfe und Manifeste der Architektur und des Städtebaus als Teile einer Ideengeschichte, welche auch in der Stadtforschung eine Rolle spielen.

Die Entdeckung der Stadt oder der Urbanität als Thema fand aber in der Architektur und im Städtebau schon viel früher statt - damit meine ich jetzt nicht nur, dass sich Bauen und Planen ja ohnehin ,schon immer' im städtischen Kontext abgespielt haben, sondern die Problematisierung von Urbanität beziehungsweise Stadt in der Architektur und im Städtebau. Letztlich waren Fragen von Herrschaft und sozialer Ordnung sowie deren Repräsentation oder das Verhältnis von Öffentlichkeit und Privatheit auch schon für antike Baumeister von Bedeutung, allerdings sind bis aufVitruvs Zehn Bücher über die Architektur, die sich hauptsächlich baulichen und ingenieurstechnischen Fragen widmen, recht wenige Schriften überliefert. Deshalb mache ich lieber einen Sprung und komme zurück zur sehr unterschiedlichen Bewertung der Großstadt, von der NiS gerade gesprochen hat. Eine sehr wichtige Strömung war zum Beispiel die künstlerisch geprägte Kritik der industrialisierten Stadt, für die Camillo Sittes (1843-1903) Buch Der Städtebau nach seinen künstlerischen Grundsätzen von 1889 exemplarisch ist (und bis heute noch - oder wieder - als eine der grundlegenden Referenzen gilt). Im Gegensatz zur Moderne beziehungsweise zum Funktionalismus ist Sittes Buch aber weniger an den Produktionsbedingungen der industrialisierten Großstadt interessiert - für Sitte war Städtebau Kunst und kein technisches oder soziales Problem.

Ganz anders wurde die industrialisierte Großstadt im Kontext des modernen und funktionalistischen Städtebaus beziehungsweise der Architektur bewertet: Einerseits sind diese ja von einer großen Faszination für die Möglichkeiten der Industrialisierung geprägt, gerade wenn es um Bautechnik geht, zum Beispiel zum Bau von Wohnungen. Die Lösung der Wohnungsfrage etwa rückte durch den industrialisierten Massenwohnungsbau in greifbare Nähe. Andererseits sind sie von einer Ablehnung der schlechten Lebensund Arbeitsbedingungen in der industrialisierten Großstadt geprägt. Die Charta von Athen, die auf dem Congrès International d'Architecture Moderne (CIAM, Internationaler Kongress für neues Bauen) 1933 vorgestellt wurde, fasst die Grundannahmen des modernen funktionalistischen Ansatzes gut zusammen. Dabei kommen neben den normativen Fragen, wie die neue, moderne Stadt gestaltet sein sollte, wie die funktionale Trennung von Wohnen, Arbeiten und Erholung zu bewerkstelligen sei, auch Fragen nach den Ursachen der Misere in der industrialisierten Stadt, wie Spekulation im Wohnungsbau, zum Ausdruck. Der Modernisierungsanspruch war gleichzeitig ein sozialreformerischer Anspruch, den man allerdings vielleicht nicht so ohne Weiteres aus den diversen Idealstadtentwürfen dieser Zeit (Le Corbusier, Hilbersheimer) oder deren Umsetzungen herauslesen kann. Letztlich hat sich die diesen Entwürfen zugrunde liegende Annahme, dass sich diese Ziele wirklich allein durch Planen und Bauen realisieren lassen würden, auch schlicht als zu kurz gegriffen herausgestellt.

Interessanterweise haben sich in der Beschäftigung mit der Urbanität und der Stadt in der Architektur und im Städtebau sowohl die modernen und 
funktionalistischen Ansätze als auch der stadtbaukünstlerische Ansatz über eine ganze Zeit hinweg in unterschiedlicher Intensität gehalten.

Stefan Höhne (SH): Für die historische Stadtforschung stellt sich die Entwicklung etwas anders dar. So lässt sich guten Gewissens behaupten, dass die Geschichte der Städte schon seit jeher ein zentrales Interesse der Geschichtswissenschaft war, und zwar schon weit vor ihrer Institutionalisierung an den Universitäten im späten 19. Jahrhundert. Als eine der ersten kulturhistorischen Arbeiten zur Stadt im engeren Sinne kann sicher die Schrift Die Kultur der Renaissance in Italien des Schweizer Historikers Jacob Christoph Burckhardt aus dem Jahre 1860 gelten. Dabei beobachtet Burckhardt in den Renaissancestädten Oberitaliens unter anderem bereits die Entstehung des modernen individuellen Subjekts, begünstigt durch eine hochgradige Arbeitsteilung, den Aufschwung merkantilistischer Ökonomien sowie die Dichte und Heterogenität der urbanen Bevölkerung. Damit macht er sozusagen ein stadtsoziologisches Argument avant la lettre. Wenn es für Burkhardt vor allem die Städte sind, welche die Emergenz moderner Subjektivität und autonomer Individualität begünstigen (und damit u. a. auch die moderne Mode erfinden), wird hier bereits ein Evergreen der Stadtforschung thematisch, der u. a. durch Weber und Simmel auch Eingang in die Soziologie gefunden und bis heute Konjunktur hat.

Jenseits solch rar gesäter Vorreiterstudien von Burckhardt oder auch von Karl Bücher wurde die deutschsprachige Stadtgeschichte um 1900 jedoch weitestgehend von Laienhistorikern oder Archivaren betrieben und war dementsprechend stark auf die Lokalgeschichte konzentriert. Auch an den Universitäten war sie mehr oder weniger marginal und wurde vor allem im Kontext von Bevölkerungswissenschaft, Rechtsgeschichte, Nationalökonomie oder der frühen Soziologie wirksam. In diesen Arbeiten werden, ähnlich wie auch in der frühen Soziologe und Volkskunde, immer wieder großstadtfeindliche Ressentiments laut, welche die ,Unnatur' und ,Maßlosigkeit ' der Metropolen geißeln und die sich dort versammelnden, potentiell revolutionären Massen kritisch beäugen. Man könnte behaupten, dass sich da also bereits eine Form von kritischer Stadtgeschichte abzeichnet, allerdings aus einer konservativ-reaktionären Richtung.

Boris Michel (BM): Bei der Geographie ist das wiederum ein wenig anders. Die Geographie ist als eine Wissenschaft entstanden, für die Städte nicht im Zentrum ihres Interesses standen. Die eben von euch angesprochene Bedeutung der Industrialisierung für ein neues Denken über Raum wurde in der Geographie lange Zeit verdrängt. Das ist sicherlich sowohl ihrer besonderen Stellung zwischen Naturwissenschaften und Sozial-/Kultur-/ Geisteswissenschaften geschuldet, als auch der Verbindung mit Entdeckungsreisen und Kolonialismus. Ihr Fokus war die Abhängigkeit des Menschen von der Natur beziehungsweise das Verhältnis von Natur und Kultur sowie die Erforschung nicht-moderner, ,primitiver und ,unzivilisierter Räume. Für Städte war da wenig Platz.

In den $1890 e r$ Jahren begannen Geographen wie Friedrich Ratzel und Otto Schlüter von Siedlungsgeographie zu schreiben. Dabei interessierten sie sich insbesondere für Lage und Form der Städte. Dass Städte sozial differenziert, 
widersprüchlich und politisch sind, war für die Geographie, die in Kategorien wie Völkern und ,dem Menschen' dachte, natürlich nicht sichtbar. Differenz wurde eher zwischen Städten betont und diese Unterschiedlichkeit von Städten als Ausdruck von so etwas wie ,Kulturkreisen', der naturräumlichen Bedingungen oder individueller Eigenlogik gesehen.

1903 erschien von Friedrich Ratzel ein Text, der sich mit der Geographischen Lage der großen Städte beschäftigte, interessanterweise genau in der Publikation, in der auch Georg Simmels Die Großstädte und das Geistesleben erstmals veröffentlicht wurde. Und ich glaube, wenig macht die Ungleichzeitigkeit dieser beiden Disziplinen in Bezug auf ein Verständnis moderner kapitalistischer Gesellschaften deutlicher. Bei Ratzel ist Stadt eine irgendwie universelle Größe, in der halt irgendwie Menschen, Verkehr und Wirtschaft verdichtet sind. Daher tauchen bei ihm dann auch relativ gleichberechtigt Konstantinopel, das antike Theben und Stuttgart auf. Dahingegen ist die Großstadt bei Simmel ein Ort der Verdichtung einer ganz spezifischen, modern-kapitalistischen Erfahrung. Und das drückt meines Erachtens doch sehr unterschiedliche Vorstellungen und Funktionen von Stadt aus. Für diese Geographie war das eine Form von Raum unter anderen und ihr Interesse war räumlich und nicht sozial ausgerichtet.

Der Begriff der Stadtgeographie setzt sich gegenüber dem der Siedlungsgeographie erst nach dem Ersten Weltkrieg durch, und in dieser Zeit entstehen zahlreiche stadtgeographische Monographien im Stil geographischer Länderkunden. Das heißt: monographische Schilderungen einzelner Städte, die idealerweise zunächst mit einer physisch geographischen Beschreibung des Naturraums einsteigen, dann eine historische Genese nachzeichnen und schließlich zu einer morphologischen Beschreibung des heutigen ,Stadtkörpers kommen. So enthält die erste Stadtgeographie Berlins sowohl Erörterungen über Relief und Niederschlag wie auch eine Kartierung der Kneipendichte. Wegen der stark idiographischen Ausrichtung dieser Geographie werden diese Städte als räumliche Individuen begriffen und stehen zunächst für nichts außer sich selbst. Eine Fragestellung oder ein Problem sucht man meist vergebens. Es geht eher um ein Sammeln von Städten. Was besonders auffällt, ist der Fokus auf Städte, die weder damals noch heute als Großstädte gelten können. Eher interessierten Klein- und Mittelstädte. Die erste Arbeit über Berlin wurde erst 1933 veröffentlicht. Eine Konzentration auf Großstädte wie bei Autoren der Chicago School, aber auch bei Simmel, Benjamin, Marx und so weiter war dieser Geographie fremd. Eine Kleinstadt in Thüringen war nicht mehr und nicht weniger interessant und bedeutsam als New York.

NiS: Auch in den soziologischen Gemeindestudien, die zwischen den Weltkriegen und dann wieder seit den $1950 e r$ Jahren zahlreich durchgeführt wurden (zu Darmstadt 1957, Euskirchen 1958, Steinfeld 1958, Dortmund 1959, Wolfsburg mehrfach seit 1959), wurden Groß- ebenso wie Kleinstädte erforscht. Anders als in den geographischen Beschreibungen ging es in den Studien der 5oer Jahre weniger um die äußere Form der Stadtgebilde als darum, das soziale Geflecht als eine Totalität zu betrachten, die für sich steht und in der sich alles, was in der Gesellschaft vorhanden ist, abbildet. Dieser Ansatz war in der Soziologie allerdings umstritten, auch wegen seines deskriptiven Charakters, und geriet zunehmend ins Abseits. 
Ganz anders ist die frühe Studie der Arbeitsgruppe um Jahoda, Lazarsfeld und Zeisel aus den 1930er Jahren zu Marienthal ausgerichtet. Dieser umfangreichen Forschung, die quantitative mit kreativ entwickelten qualitativen Methoden verbindet, geht es zwar um den Alltag in der Siedlung Marienthal, einem kleinen niederösterreichischen Ort südlich von Wien, jedoch ohne dass der gesamtgesellschaftliche Kontext, in dem dieser Gegenstand steht, ausgeblendet würde. Die Studie interessiert sich in erster Linie für die Auswirkungen der zu dieser Zeit neu aufgetauchten Massenarbeitslosigkeit, für die subjektive Stimmungslage, die Alltagsbewältigung sowie die materielle Versorgung der Bewohner_innen. Wie im Labor wird dies am Beispiel einer Siedlung mit extrem hohem Arbeitslosenanteil untersucht. Dafür haben die Forschenden, die einige Monate mit in der Siedlung gelebt haben, auch über verschiedene, von ihnen organisierte Aktivitäten wie medizinische Sprechstunden, Kurse, Kinderwettbewerbe und Kleidersammlungen den Zugang zu den Marienthaler_innen gesucht. Das Forschen hatte also auch einen karitativen Anteil - den Beforschten sollte offenbar etwas zurückgegeben werden, wie man heute sagen würde. Ja, sicherlich waren die Interessen und Forschungszugänge dieser soziologischen Studie ganz anders als die anderer Disziplinen. Sie ist vielleicht beispielhaft für die soziologische Ausrichtung in der Zwischenkriegszeit, wenn sie auch wegen ihrer aufwändigen Forschungsarbeit außergewöhnlich ist und erst mit dem neuen Interesse an qualitativer Forschung in der Soziologie seit den 1980er Jahren wieder stärker rezipiert wird.

BM: Ja, genau. In der Geographie hatte der Blick auf Kleinstädte eine andere Qualität. Ich würde das auch eher erkenntnistheoretisch begründen als forschungspraktisch oder empirisch, wie ich es für die Soziologie vermuten würde. Ein wichtiger Grund war meines Erachtens der, dass die Geographie vielfach von der Idee getrieben war, Natur und Mensch müssten in ihrem Wechselverhältnis beschrieben werden, meist sogar als Prägung des Menschen durch die Natur. Die Großstadt galt da als etwas zu Künstliches, etwas, das die Natur zu sehr verdrängte. Da wundert es sicherlich auch nicht, dass die Geographie vielfach stadtkritische Momente aufweist. Das verschärft sich ab Ende der 1920er und insbesondere in Verbindung mit dem Zweig der Geopolitik.

$\boldsymbol{S H}$ : Schaut man sich die Entwicklung der nicht nur deutschsprachigen stadthistorischen Forschung seit Beginn des 20. Jahrhunderts an, so lassen sich die dort entstehenden Forschungen vielleicht grob in drei Kategorien einteilen. Zunächst finden sich unzählige lokale Stadtgeschichten, die sich der Entwicklung einzelner Städte widmen und dabei oftmals auch von, Laienhistorikern' oder Chronisten verfasst werden. Hier widmet man sich vor allem der rechtlichen Entstehung von Stadtverfassungen und adressiert kaum die realen sozialen und wirtschaftlichen Lebenswirklichkeiten was der Geschichtswissenschaft bereits um 1900 viel Kritik einbringt, beispielsweise von Werner Sombart und Georg Simmel. Allerdings finden sich dann vor allem ab den 1920ern auch vermehrt Studien, die Teilprozesse städtischer Entwicklung und auch soziale Dimensionen in den Blick nehmen, wie beispielsweise Wohnen, Städtebau oder Migration. Diese Studien stellen 
zugleich wichtige Impulse für die Herausbildung der Sozialgeschichte dar, nicht zuletzt in Deutschland und Frankreich.

Drittens formiert sich in diesem Zeitraum auch eine historische Urbanisierungsforschung, welche jenseits von Lokalgeschichte die Entwicklung der Städte als Ausdruck gesamtgesellschaftlichen Wandels adressiert. Sie versucht dann Urbanisierungsgeschichte als große Erzählung im Sinne einer Longue Durée zu entfalten, die ganze Hemisphären und Epochen umfassen kann. Dabei finden sich gerade in letztgenannter Kategorie immer wieder wirklich beeindruckende Arbeiten, die auch an konzeptionell-theoretischen Debatten und Zugängen interessiert sind. Spätestens ab den 1960ern zeigt sich dies unter anderem auch an einem vermehrten Interesse an stadtsoziologischen und -geographischen Zugängen; ab den 1980ern wird beispielsweise Lefebvre auch in der Stadtgeschichte ansatzweise rezipiert. Dies kann man als Ausdruck einer produktiven Unsicherheit dieser Disziplin über den konzeptionellen Status des Untersuchungsgegenstands ,Stadt' deuten, die die ,Urban Studies' heute ja generell auszuzeichnen scheint. Auch in der historischen Urbanisierungsforschung wird sie immer wieder thematisch und mündet regelmäßig in neue Debatten und Definitionsangebote um Begriffe wie ,Urbanität‘ und ,Verstädterung sowie deren Rollen im Verhältnis zu Großprozessen von (De-)Industrialisierung, Modernisierung, Durchsetzung des Fordismus et cetera. Dabei werden auch vermehrt geographische wie stadtsoziologische Arbeiten herangezogen. Damit wäre diese Forschung durchaus auch an Debatten anschlussfähig wie sie unter anderem in $\mathbf{~ u ~} \mathbf{b} \backslash \mathbf{u} \mathbf{r} \mathbf{b}$ a $\mathbf{n}$ geführt werden. Doch ich greife vor; wir könnten vielleicht noch genauer auf die Rolle der deutschsprachigen Stadtforschung in der ersten Hälfte des 20. Jahrhunderts eingehen, oder?

$\mathbf{s} \backslash \mathbf{u}$ : Zur Rolle der Disziplinen im Nationalsozialismus würde ich gerne mehr hören. Etwa zu der Rolle von so jemandem wie Andreas Walther, der in den 1930er und 1940er Jahren seine Erfahrungen mit der Chicago School in eine NS-Stadtsoziologie übersetzt hat. Auch die Geographie ist nicht gerade für ihre antifaschistische Geschichte bekannt.

NiS: In den 1920er Jahren hat sich der Soziologe Andreas Walther nach einem Forschungsaufenthalt in Chicago insbesondere an der forschungspraktischen Ausrichtung der Chicagoer Studien orientiert. Während es Robert E. Park in Chicago jedoch um ein unvoreingenommenes Verstehen der städtischen Vorgänge ging, hatte Walther im Sinn, durch seine Studien Einfluss auf seinen Forschungsgegenstand zu nehmen. Als Hamburger Soziologieprofessor kartographierten er und seine Studierenden in der Zeit des Nationalsozialismus in Hamburg unter anderem in seiner 1934 von der DFG geförderten Studie Notarbeit 51 „gemeinschädigende Regionen“beziehungsweise „soziale Krankheitsherde“ der Stadt. Darunter verstand er unangepasste Lebensformen, Verhaltensweisen und politische Orientierungen ebenso wie körperliche Einschränkungen, die er allesamt mit Minderwertigkeit, Kriminalität und Asozialität assoziierte. Aufgrund der angeblich „volksschädigenden Wirkung“ der Stadtgebiete, in denen er eine Häufung entsprechender Lebensweisen festgestellt hatte, empfahl Walther deren „Sanierung“ und bereitete damit die brutalen nationalsozialistischen bevölkerungspolitischen Eingriffe mit vor. 
Walther hat also die Vorgehensweisen der Chicago School übernommen, sich aber in seiner Forschung an politischen Zielen und praktischer Anwendbarkeit der Ergebnisse orientiert. Dies lässt sich ausführlich unter anderem in Ulrike Kändlers gerade erschienener Studie Entdeckung des Urbanen nachlesen.

$\boldsymbol{B M}$ : Das, was ich vorhin als stadtkritische Momente der Geographie zwischen dem Ersten und Zweiten Weltkrieg angedeutet habe, radikalisiert sich in den 1930er Jahren bei einigen Autoren. Insbesondere ein radikaler Antisemit wie Siegfried Passarge fällt mir dazu ein, aber auch die Linie der Geopolitik von Karl Haushofer, die in der Verstädterung die Gefahr einer „Entwehrung“ des Ländlichen gesehen hat. Für beide ist Verstädterung die Materialisierung und Verschärfung einer Erzählung von gesellschaftlichem Niedergang, ein bisschen im Sinne von Oswald Spenglers Untergang des Abendlandes sicherlich. Städte, beziehungsweise die Menschen in Städten, gelten diesen Autoren als ,wurzellos', liberal und vergeistigt. Und die großen Städte sind dann auch noch der Sitz des Judentums, des internationalistischen Proletariats und des Finanzkapitals. Nationalsozialistische Geographie müsse hingegen zur Verwurzelung des Menschen mit der Scholle und nationaler Gesinnung beitragen. Die relativ konservative und oftmals reaktionäre Grundhaltung in der Geographie (man wollte seine Kolonien zurück, sah in den Grenzen von Versailles ein widernatürliches Unrecht, hatte wenig Sympathie für die Arbeiter_innenbewegung usw.) machte die Disziplin bekanntermaßen recht offen für den NS.

Zugleich beginnt hier erstmals das, was man ,angewandte Geographie nennen könnte, also eine Raumplanung, die eben auch Städte plant. Walter Christaller fällt mir dazu ein, der seine Theorie der ,Zentralen Orte' im Rahmen des ,Generalplans Ost' in die Praxis umsetzen sollte. Auch zeigt man sich interessiert an der Verländlichungspolitik von Mussolini und an Fragen nach biopolitisch optimalen Siedlungsformen. Dass ein Geograph sich allerdings zu Albert Speers ,Germania', quasi nationalsozialistischer Metropolenpolitik, geäußert hätte, wäre mir unbekannt.

SH: Im Gegensatz zur Geographie und Soziologie scheint die deutschsprachige historische Urbanisierungsforschung der 1920er und 1930er offenbar kaum einflussreiche Arbeiten hervorgebracht zu haben. Stattdessen war es nun vor allem die aufstrebende Bevölkerungswissenschaft, die sich der Stadtentwicklung und urbanen Migration in Mitteleuropa historisch näherte. Diese Forschungen wurden dann bezeichnenderweise auch während des Nationalsozialismus weitergeführt. Was die Funktion der Stadtgeschichte als Disziplin im engeren Sinne während des Nationalsozialismus angeht, so scheint es dazu bislang wohl kaum dezidiert Forschungen zu geben. Zwar ist mittlerweile gut ausgearbeitet, dass die deutsche Geschichtswissenschaft vor allem ab 1939 intensiv darum bemüht war, eine der nationalsozialistischen Expansions- und Vernichtungspolitik dienliche Geschichtsinterpretation voranzutreiben. Zumindest nach meinem Kenntnisstand hat die Stadtgeschichte darin aber wohl kaum eine wichtige Rolle gespielt. Dies könnte vielleicht auch daran liegen, dass sie oftmals Narrative mobilisiert, die nicht auf klassisch historische Akteure wie Nation, Herrscher oder Volk zentriert sind, sondern eben eher auf Städte und ihre Bewohner_innen. Dennoch würde es sich 
lohnen, da noch mal genauer hinzuschauen und beispielsweise die Rolle von Stadthistoriker_innen im Kontext der sogenannten ,Ostforschung' genauer zu beleuchten. Allerdings gab es während des Nationalsozialismus natürlich eine Vielzahl von Arbeiten zur Stadt, die eine starke historische Dimension aufwiesen, auch wenn diese vor allem von Rasseforschern und völkischen Anthropologen verfasst wurden. Sie tragen dann so sprechende Titel wie Die Verstädterung - Ihre Gefahren für Volk und Staat oder Großstadt und Volkstum. Darin wird die bereits weit verbreitete Großstadtkritik sowohl zunehmend hysterisiert und apokalyptisch zugespitzt wie auch rassenbiologisch fundiert - ein wahres Gruselkabinett der Stadtforschung.

Zudem kann man sagen, dass nicht nur die Disziplinengeschichte der Stadtgeschichte im Nationalsozialismus bislang kaum beforscht ist, sondern auch die Stadtentwicklung dieser Zeit erstaunlich wenig Beachtung gefunden hat, wenn man von einzelnen Studien beispielsweise zu Trier oder Hildesheim wie auch den unzähligen Publikationen über den Dauerbrenner ,Germania' mal absieht. Hier gäbe es sicher noch einiges zu entdecken.

NG: In der Architektur und im Städtebau hat Werner Durth mit Deutsche Architekten: Biographische Verflechtungen 1900-1970 anhand der eher bekannten Protagonisten (soweit ich mich erinnere, war keine einzige Frau darunter) nachvollzogen, von welchen erstaunlichen Kontinuitäten die Disziplinen geprägt waren, weit über den Nationalsozialismus hinaus. Eine ganze Reihe der modernen Avantgarde wanderte zwar aus, viele praktizierten aber einfach weiter, teilweise mit entsprechend anderen ästhetischen Grundsätzen. Eigentlich wollte ich aber noch kurz einen der Kristallisationspunkte im Richtungsstreit kurz vor dem Nationalsozialismus erwähnen: die Stuttgarter Weissenhofsiedlung, die als Werkbund-Ausstellung 1927 in Stuttgart gebaut wurde. Die wichtigsten Vertreter des Neuen Bauens waren daran beteiligt und die Leitung hatte Mies van der Rohe inne. Die Siedlung besteht hauptsächlich aus weißen, modernen Gebäuden mit Flachdächern. Sie wurde schnell von einem Großteil der traditionalistischen ,Stuttgarter Schule‘ um Paul Schmitthenner und Paul Bonatz abgelehnt und mit ,Araberdorf tituliert. Als Gegenentwurf bauten die Stuttgarter Architekten 1933 in Blickweite die Kochenhofsiedlung, weitgehend im Holzbau und mit Satteldächern. Die Kochenhofsiedlung entstand im Rahmen der Ausstellung „Deutsches Holz für Hausbau und Wohnung“. Der oft auch als ,internationaler Stil' bezeichneten Moderne wurde in Stuttgart der Heimatstil entgegengestellt - das sind zwar keine dezidierten Forschungsprojekte, für mich passen sie aber dennoch als Ausdruck einer materialisierten Debatte in unser Gespräch.

NiS: Für die 1950er Jahre konstatiert Ulfert Herlyn eine ,statische‘ Soziologie, deren Fokus auf der Dokumentation der Stabilität sozialer Systeme während der gesellschaftlichen Reorganisation und des Wiederaufbaus der Städte in der BRD entsprechend ,alter, traditionsreicher Muster“ lag, und die von der Stadtforschung zustimmend begleitet wurde. Herrmann Korte attestiert der Soziologie dieser Zeit ein starkes Bedürfnis nach Stabilisierung und Harmonisierung, was sich auch in den bereits erwähnten Gemeindestudien zeigt, die sich allerdings wiederum auch stark an den US-amerikanischen community studies orientieren. Für die 1960er Jahre lässt sich dann ein 
Wirtschaftsprimat feststellen, das sich stark an einem Fortschrittsglauben und einem umfassenden Modernisierungsschub festmacht, begleitet von einer beschleunigten Verstädterung. Interessant ist die Fragerichtung damals, in der es darum ging, wie die Großstadt den wachsenden Anforderungen der Planungspraxis gerecht werden könnte, wie es Herlyn formuliert. Insbesondere für den Wohnungs- und Städtebau wurden von der Soziologie lösungsrelevante Antworten erwartet. Die Disziplinen Soziologie und Städtebau rückten also in dieser Zeit enger zusammen, was auch Hans-Paul Bahrdts Abhandlung Die moderne Großstadt. Soziologische Überlegungen zum Städtebau von 1969 deutlich macht. In ihrer Kooperation mit dem Städtebau wurde die Soziologie einerseits zu dessen Hilfswissenschaft, andererseits versuchten Soziolog_innen aber auch, den Städtebauer_innen und Planer_innen die Stadt als soziales System näherzubringen. Hier setzt also die Stadtsoziologie ihre Funktion als Zulieferin für Stadtplanung und -politik fort, allerdings im Gegensatz zur NS-Zeit ohne deren menschenverachtenden politischen Eifer.

NG: Im Städtebau (beziehungsweise an den Architekturfakultäten - damals) kann man Mitte der 1960er Jahre ähnliche Tendenzen beobachten: Die Studierenden begannen eine Verwissenschaftlichung von Architektur und Städtebau zu fordern. Entwürfe sollten anhand ,harter Kriterien bewertbar und begründbar werden - die Bedarfsplanung stand hoch im Kurs, ebenso Systemtheorie und Kybernetik. Und es gab zu dieser Zeit eine unglaubliche fachliche Ausweitung im Städtebau: Neben der Öffnung in Richtung Sozialwissenschaften wurde auch die Öffnung in Richtung der wirtschaftlichen und technischen Wissenschaften wie Ökonomie und Baustoffkunde gefordert und teils dann auch erzielt. Sozialpsychologie und Pädagogik wurden für einige auch zum Thema. Zugleich verbanden viele große Hoffnungen mit der Industrialisierung, was die Überwindung der Wohnungsnot angeht. Einige nahmen in diesem Zusammenhang auch Verbindung auf mit Kolleg_innen in der DDR, die die Rationalisierung und Standardisierung etwas radikaler vorantreiben konnten.

NiS: In diese Zeit fällt ja auch die Gründung der Stadt- und Raumplanungsfakultäten mit starker sozialwissenschaftlicher und interdisziplinärer Verankerung, zum Beispiel in Dortmund 1969 und an der TU Berlin 1972. Durch ihre enge Zusammenarbeit mit dem Städtebau hat die Stadtsoziologie in den 196oer Jahren entscheidend zum funktionalistischen Umbau der Stadt beigetragen. Sie arbeitete ihm zu, indem sie ihm beispielsweise auf der Grundlage großer Sozialerhebungen in Sanierungsgebieten Sozialdaten für die Bedarfsermittlung lieferte, aufgemacht in formalisierten Darstellungsformen. Ihre Unterstützung half, vorzeitig mögliche Konflikte zu verringern und das bestehende System zu optimieren und so abzusichern, dass die Städtebauer_innen ihre technisch-ästhetischen Optimierungsversuche leichter durchsetzen konnten. Dass dies ab einem bestimmten Zeitpunkt immer weniger gelang, zeigen die Proteste seit den 1960er Jahren, die sich gegen Kahlschlagsanierung in vielen Städten regten.

NG: Vor der von dir beschriebenen Abspaltung der Stadt- und Regionalplanung von der Architektur gab es ja auch viel Zusammenarbeit von Architekt_innen 
sowie Städtebauer_innen und Soziolog_innen (und teils auch Pädagog_innen). Ich denke zum Beispiel an das damals noch recht frische Format der Stadtteilarbeit, mit dem Studierende der Berliner Universitäten, vor allem jedoch die Architekturstudent_innen der TU Berlin, ab 1968 in diversen Basisgruppen zu experimentieren begannen. Aus einer Kritik an der Kahlschlagsanierung in Kreuzberg und dem Bau des Märkischen Viertels, die sich Ende der 1960er formierte, haben sich verschiedene Gruppen von Studierenden sowohl in Kreuzberg als auch im Märkischen Viertel in der Stadtteilarbeit engagiert und Bewohner_innen beraten beziehungweise sogenannte Gegenplanungen entwickelt. In Kreuzberg beispielsweise gründeten Architekturstudierende und ein Soziologe der Freien Universität 1968 gemeinsam das Büro für Stadtsanierung und Soziale Arbeit, das neben der Stadtteilarbeit auch eine der wichtigsten Publikationen zu dieser Arbeit herausgab: Das Buch Sanierung für Wen?. Neben der Kritik an den Abrissen der Altbauten hinterfragten die Studierenden auch zunehmend den modernen und funktionalistischen Massenwohnungsbau in Westberlin, einerseits in Bezug auf die Finanzierung und politische Verfilzungen, andererseits aber auch in Bezug auf die sozialen und psychologischen Folgen von Monotonie und Entfremdung.

Insgesamt kamen zu dieser Zeit eine Menge spannender Bücher heraus, die diese große Offenheit über disziplinäre Grenzen hinweg zeigen und in der - vielleicht aus heutiger Sicht relativ überraschend - neben den Sozialwissenschaftler_innen auch eine Gruppejunger Architekt_innen und Städtebauer_innen (wovon einige später in den sich kurz darauf gegründeten Planungswissenschaften arbeiten werden) kräftig mitwirkte. Ich denke dabei zum Beispiel auch an das Kursbuch Nr. 27: Planen Bauen Wohnen oder den von Hans G. Helms und Jörn Janssen herausgegebenen Band Kapitalistischer Städtebau. Die Bezüge waren vielfältig und reichten von der kritischen Theorie über Sozialpsychologie bis hin zur Semiotik.

$\boldsymbol{S H}$ : Die späten 1960er und frühen 1970er Jahre sind bemerkenswerterweise auch die Jahre, in denen sich im deutschsprachigen Raum eine eigenständige Stadtgeschichte der Moderne formiert. Sie tritt mit einem deutlichen Geltungsanspruch aus dem Schatten einer generellen Geschichtswissenschaft heraus. In diesem Bestreben kommt vor allem der 1970 ins Leben gerufenen Fachzeitschrift Informationen zur modernen Stadtgeschichte (IMS) eine zentrale Bedeutung zu. Bis heute erscheint sie mehrmals jährlich und bildet mit mittlerweile mehr als 90 Ausgaben ein beeindruckendes Kompendium stadthistorischer Forschung in West-Deutschland. Schaut man in die allerersten noch sehr dünnen und mit Schreibmaschine verfassten Ausgaben, so wird dort bereits eine Krise der Geschichtswissenschaften allgemein und der Stadtgeschichte im Besonderen beklagt, die sich nicht zuletzt in unzähligen „Bildbände[n] mit belanglosen Texten“ niederschlagen würde. Dass man stattdessen eine ganz andere historische Stadtforschung wollte, wird bereits im programmatischen Geleitwort der ersten Ausgabe deutlich: Hier postuliert der Historiker Hans Herzfeld den Anspruch einer Stadtgeschichte der Moderne als „Geschichte eines umfassenden Urbanisierungsprozesses“, die weit mehr als nur Lokalgeschichte oder die isolierende historische Analyse einzelner Städte sein will. Es ging ab jetzt also eher um die Geschichte der Urbanisierung als um klassische Stadtgeschichte im Sinne 
einer Lokalgeschichte. Dieses sympathische Programm, die Entwicklung von Städten im 19. und 20. Jahrhundert als Teil gesamtgesellschaftlicher Transformationen (wie bspw. der Industrialisierung oder Modernisierung) zu verstehen, hat sich als sehr erfolgreiche und produktive Strategie erwiesen.

Ein nicht unähnliches Vorgehen verfolgte auch die durchaus produktive Stadtgeschichtsforschung in der DDR, die auf Grundlage des Historischen Materialismus die Städte als Orte sozialer Kämpfe und Klassenkonflikte adressiert. Dass es hier einen starken Fokus auf die mittelalterliche Stadtkultur gab, liegt nicht zuletzt in der marxistischen Geschichtstheorie selbst begründet. So galt schon für Marx und Engels die Blüte der europäischen Städte als Indiz für den Fortschritt der Feudalgesellschaft gegenüber der Antike. Allerdings avancierte mit der Entstehung bürgerlicher Ordnungen der Nationalstaat zur zentralen politischen Instanz. Nach dieser Deutung waren die modernen Städte bestenfalls noch Schauplatz dieses historischen Prozesses, was sich auch in dem weitgehenden Desinteresse der DDR-Historiker_innen an der modernen Stadtgeschichte niederschlug. Dies ist jedenfalls mein Eindruck - eine genauere Untersuchung der historischen Urbanisierungsforschung in der DDR steht noch aus und verspräche sicher interessante Erkenntnisse zu Grenzen und Perspektiven klassisch-marxistischer Stadtforschung.

Interessant in diesem Zusammenhang ist übrigens auch eine Forschungsrichtung namens ,New Urban History', die primär im englischsprachigen Raum um 1970 einen ebenso kurzen wie spektakulären Aufschwung erlebte. Imprägniert vom Geist der qualitativen Revolution versuchten hier Historiker_innen mit Hilfe neuer quantitativer Verfahren und soziologischer Theorieimporte die Alltagserfahrungen der einfachen Stadtbewohner_innen historisch zu rekonstruieren. Dieser ziemlich interessante makroperspektivische Ansatz hatte jedoch auf die deutschsprachige Forschung bestenfalls marginalen Einfluss und ist auch in den USA und in Großbritannien mittlerweile nahezu vollständig verschwunden. Vielleicht erlebt er ja durch den aktuellen Hype um die digitalen Geschichtswissenschaften und Big Data eine Renaissance.

$\boldsymbol{B M}$ : Die 1950er Jahre waren wir in der Geographie - vielleicht so, wie das gerade für die Soziologie beschrieben wurde - ziemlich statisch und vom Versuch geprägt, an das alte Paradigma anzuschließen, sich von einer Verwicklung mit dem Nationalsozialismus zu befreien (bzw. zu begründen, warum man nie etwas damit zu tun hatte) und die Perspektive ein wenig durch eine funktionalistische Sprache zu modernisieren. Dabei wurden vermutlich auch erstmals Texte von Soziolog_innen gelesen. Es gab also erste Versuche, die Geographie als eine Sozialwissenschaft zu denken, und dabei spielten städtische Themen durchaus eine wichtige Rolle.

Die späten 1960er Jahre waren auch in der Geographie eine zentrale Phase für die umfangreichere Modernisierung der Disziplin. Dabei ist der Begriff ,Modernisierung' sicherlich treffender als hier in der deutschsprachigen Geographie von einer kritischen Wende zu sprechen. In den späten 1960er Jahren wurden auch in der Geographie die gesellschaftlichen Konflikte dieser Zeit deutlich. Dabei spielten sowohl inneruniversitäre wie außeruniversitäre Momente eine Rolle, sei es der kulturelle Wandel oder die 
Krise des Bildungssystems. Man sollte nicht unterschätzen, welche Rolle die Einführung der Diplomstudiengänge seit den frühen 1960er Jahren gespielt hat. Die Geographie befand sich sicherlich schon vorher in einer Identitätsund Relevanzkrise und hatte ihre Legitimation zunehmend in der Lehrer_innenausbildung gesucht. In den 1960er Jahren gab es dann eine Hinwendung zu quantitativen Verfahren und eine Stärkung der angewandten Geographie. Gerade von studentischer Seite wurde die Frage der Relevanz, der Praxis und so weiter diskutiert. Um welche Praxis und welche Relevanz es dabei ging, war natürlich umkämpft: um Stadt- und Regionalplanung zur Verbesserung der Bindungen der Kapitalakkumulation im fordistischen Staat oder um die Formulierung von linken Alternativen. Aber zumindest aus Anekdoten und Berichten wird klar, dass auch Geograph_innen mitbekamen, was in den späten 1960er Jahren in der BRD geschah.

NiS: Die Proteste und Bewegungen Ende der 1960er Jahre hatten offenbar auch großen Einfluss auf die Stadtsoziologie und haben einen Paradigmenwechsel herbeigeführt. Die Stadtsoziologie gewinnt an politischer Relevanz: Community power avanciert zu einem wichtigen Forschungsgegenstand, wobei die als eindimensional empfundene Ästhetik des städtebaulichen Funktionalismus und die Zergliederung und Zurichtung der Städte ebenso kritisiert wird wie die damit assoziierte Ausrichtung auf kapitalistische Verwertung. Ins Zentrum des Interesses rücken dabei die Auswirkungen der baulich-räumlichen Veränderungen auf den Menschen und sein Verhalten. Alexander Mitscherlichs Kritik in Die Unwirtlichkeit unserer Städte von 1969 geht in genau diese Richtung. Er kritisiert darin die Funktionstrennung der Städte, aus der eine „Unwirtlichkeit“ resultiere, die „niederdrückend“ wirke, beklagt die Zerstörung und Zersiedlung des städtischen Umlands, die wachsende „Stadtwüste“, beklagt das Fehlen eines strukturierenden Städtebaus und den Verlust klassischer Stadtstrukturen. Hier wird also nun aus einer linken, großstadtkritischen Strömung heraus gegen ,Entfremdung' argumentiert. In den 1970er Jahren kommt es zu einer starken Ausweitung und Konsolidierung stadtsoziologischer Forschung und Lehre an Universitäten, sowohl in theoretischer als auch empirischer Hinsicht. Für den US-amerikanischen Kontext identifiziert Herbert Gans in den 1980er Jahren zwei theoretische Strömungen, die damals wichtiger wurden: die neo-marxistischen und die neo-ökologischen Analysen zu den Zusammenhängen von Kapitalismus, Stadt und Gesellschaft. Allerdings scheint es, abgesehen von Castells und Lefebvre, von denen einige Werke übersetzt wurden, damals in der BRD kaum eine Rezeption nicht deutschsprachiger kritischer stadtsoziologischer Debatten zu geben.

NG: Interessanterweise wurde Kampf in den Städten von Castells von Architekt_innen übersetzt und 1975 im VSA Verlag in der Reihe Analysen zum Planen und Bauen herausgegeben. Was die Rezeption nicht deutschsprachiger stadtsoziologischer Debatten angeht, nehme ich es etwas anders wahr: In Sanierung für Wen? (1970 vom Büro für Stadtsanierung und soziale Arbeit herausgegeben) sind zum Beispiel diverse Beiträge zu Partizipation, zu Mieterinitiativen oder Mieterstreiks aus den USA und England enthalten. Die frühen ARCH+-Hefte (damals noch „Studienhefte für architekturbezogene Umweltforschung und -planung“) bezogen für einige Jahre 
ab Heft 9 (1970) zu Themen wie advocacy planning oder Stadtteilarbeit vielfach auch nicht deutschsprachige stadtsoziologische Debatten mit ein. Dort erschien 1977 dann auch eine frühe Rezeption stadt- und raumbezogener Texte von Lefebvre, etwa Die Produktion des städtischen Raumes und ein Beitrag zur Revolution der Städte. Bei unserer Forschung für das Buch Vergessene Schulen: Architekturlehre zwischen Reform und Revolte um 1968 (das im Herbst 2016 bei Spectorbooks erscheint) hat mich die Fülle der internationalen Bezüge, die es damals schon gab, auch überrascht. Für das Buch haben wir viele Zeitzeug_innen interviewt, die damals an den Architekturfakultäten von Berlin und Stuttgart eingeschrieben waren oder arbeiteten. Die Protagonist_innen berichteten sowohl von diversen Reisen als auch von einem regen internationalen Austausch, teilweise sogar weit über Europa hinaus. Hauptsächlich wurden jedoch die verschiedenen Bewegungen verfolgt, die es in den USA, England und Frankreich gab, sei es in Bezug auf Mieterproteste, Designmethoden oder partizipative Ansätze der Planung. Einige verfolgten auch die italienische Bewegung (z. B. mit Planungsansätzen in Bologna, zu denen es relativ frühe Arbeiten von Bodenschatz und Harlander gibt).

Die von dir angesprochene Einteilung in neo-marxistische und ökologische Ansätze sehe ich in den Debatten zum Städtebau aber auch. Bei den neo-marxistischen Ansätzen gab es jedoch eine strikte Abstufung, die später auch in einer Fragmentierung der Bewegung resultiert hat: Während sich der eine Teil der Debatte um Stadtteilarbeit, Partizipation und die Verbesserung von Lebens- und Arbeitsverhältnissen rankte, widmete sich der andere Teil um die proletarische Revolution. Stadtteilarbeit et cetera wurde von den Revolutionär_innen als reformistisch bezeichnet und deren Akteure als sogenannte ,Refos ${ }^{6}$ verspottet.

NiS: In der bundesdeutschen Stadtsoziologie der 1970er und 8oer ist der sozialökologische Ansatz unter anderem mit Friedrichs, Hamm und Atteslander verbunden, später mit Häußermann/Siebel und Dangschat, die ihn zur Analyse der sozialen und räumlichen Organisation der Gesellschaft aufgreifen. Ihre Segregationsforschung beschäftigt sich nun mit der durch räumliche Trennung erzeugten sozialen Kluft zwischen den verschiedenen Klassen; die Teilhabe an sozialer Infrastruktur wird ebenso zum Thema wie die Kumulation von Benachteiligungen für bestimmte soziale Gruppen. Auch lokale Macht- und Entscheidungsstrukturen werden in die Analysen einbezogen und eine breitere Partizipation an politischen Entscheidungsprozessen vorgeschlagen. Die empirische Forschung ist weiterhin stark auf die stadtund wohnungspolitische Bedarfsermittlung ausgerichtet, was Häußermann/ Siebel 1978 stark kritisieren.

Für die 1980er Jahre stellt Herlyn ein abnehmendes Interesse für anwendungsbezogene stadtsoziologische Forschung fest, „eine tiefgreifende Skepsis gegenüber ökonomisch nicht abgesicherten bzw. ,durchgerechneten sozialwissenschaftlichen Konstrukten und Konzeptionen“. Dies wird aus Sicht der Stadtsoziologie als eine ,Krise' gedeutet. Außerdem artikulieren insbesondere feministische Forscherinnen seit den 1980er Jahren eine feministische Kritik an städtischen Strukturen und zeigen deren Zusammenhänge mit patriarchalen und heterosexuellen Ordnungen, zum Beispiel 
Becker, Dörhöfer, Rodenstein und Terlinden, später dann beispielsweise Bauhardt, Breckner, Frank, Löw, Ruhne, Sturm und andere, die organisiert sind in der Sektion Stadt- und Regionalsoziologie der DGS, in einer eigenen Arbeitsgruppe, sowie in der FOPA (Feministische Organisation von Planerinnen und Architektinnen), die 1981 in Berlin gegründet wurde. In beiden Organisationen, die Soziologie und Planung beziehungsweise Architektur zugeordnet sind, existieren personelle Überschneidungen.

$\boldsymbol{S H}$ : Dies ist ein interessantes Beispiel für unterschiedliche Konjunkturen der einzelnen Disziplinen der Stadtforschung. Wenn du für die 1980er Jahre bereits ein abnehmendes Interesse an stadtsoziologischen Forschungen konstatierst, erlebt zeitgleich die westdeutsche Urbanisierungsforschung innerhalb der Geschichtswissenschaften einen bis heute unerreichten Boom, samt eigenem Sonderforschungsbereich, Publikationsflut et cetera. Insgesamt lässt sich für die 1970er bis frühen 1990er Jahre eine beeindruckende Konjunktur der kulturhistorischen Urbanisierungsforschung in Deutschland konstatieren. Dabei war es sicher auch die Abwendung von klassischen Sujets der Geschichtswissenschaft, wie Nation und Staat, sowie die Hinwendung zu Fragen der Sozial- und Alltagsgeschichte, die dieses Interesse begünstigt haben. Diesen Schub einer Verwissenschaftlichung und Institutionalisierung der deutschsprachigen Stadtgeschichte bezeugt auch eine zweibändige Bibliographie zur deutschen historischen Städteforschung aus dem Jahre 1996, die bereits mehr als 14.00o Publikationen verzeichnen kann. Hier findet sich also eine gigantische Menge an stadthistorischem Wissen, das jedoch bis auf einzelne Ausnahmen erstaunlich wenig in anderen Feldern und Disziplinen der Stadtforschung rezipiert worden ist.

NG: Im Städtebau waren die 1980er Jahre geprägt von dem Paradigma der ,behutsamen Stadterneuerung' und ab 1975 (dem europäischen Denkmalschutzjahr) auch von Fragen der Erhaltung von Altbauquartieren. Im Grunde war das dann ja auch die Kehrtwende gegenüber den modernen und funktionalistischen Ansätzen, die ich vorhin beschrieben habe - die aber auch schon vom „Team 10“, den jungen Organisator_innen des 10. CIAM Kongresses, kritisiert wurden. Eine wichtige Referenz in Bezug auf die ,behutsame Stadterneuerung' war dabei auch Wolf Jobst Siedlers Die gemordete Stadt. Abgesang auf Putte und Straße, Platz und Baum von 1964 (vielleicht als ein Pendant zu Jane Jacobs Tod und Leben großer amerikanischer Städte von 1961, das ja auch erstaunlich früh in der DDR rezipiert wurde, z. B. von Brigitte Reimann). Neben dem sozialen Anspruch der behutsamen Stadterneuerung entwickelten sich zu dieser Zeit im Städtebau andere ästhetische Kategorien und Bezugspunkte wie die (Wieder-)Entdeckung der Europäischen Stadt und ein wachsendes Interesse an der identitätsstiftenden Kraft von Symbolen und Referenzen in der Postmoderne. Im Grunde stellt diese Hinwendung zur Postmoderne dann auch einen Abschluss der Öffnung von Architektur und Städtebau in Richtung der Sozialwissenschaften dar, die sich im Zuge der 1968er Bewegung ergeben hatte. Postmoderne Architektur und postmoderner Städtebau sollte sich wieder deutlicher auf sich selbst beziehen und zum Beispiel bauliche Traditionen zitieren und damit zum Erzählen gebracht werden. 
$\mathbf{s} \backslash \mathbf{u}:$ Jetzt haben wir über unterschiedliche disziplinäre Zugänge zur Stadt/Urbanität gesprochen. Wie kam es dann zu explizit kritischen Stadtforschungsansätzen?

NiS: Die Frage, ob und wann, also in welchen gesellschaftlichen Konstellationen, es bereits kritische soziologische Stadtforschung gab, finde ich deswegen schwer zu beantworten, weil vorher der Begriff des Kritischen geklärt werden muss. Engels (1845) hatte früh eine Gesellschaftskritik im Blick, als er die Lebens- und Wohnbedingungen der Arbeiter_innen in den Städten zum Anlass für seine Analysen nahm. Weitere empirische Studien zu den Wohn- und Lebensverhältnissen in den großen Städten in der zweiten Hälfte des 19. Jahrhunderts hatten eher sozialreformerische beziehungsweise sozialpolitische Ideen. Es ging darum, das ,Chaos zu ordnen', die hygienischen Verhältnisse in den proletarischen Wohnquartieren zu verbessern und dabei zugleich die Stadtteile überhaupt kontrollierbar zu machen. Die Entstehung der empirischen Sozialforschung ist eng mit der Entstehung von Großstädten und der Erforschung städtischer Lebensbedingungen verbunden. Diese Forschung stellte sich allerdings häufig in den Dienst der Sozialpolitik.

NG: Es macht sicherlich Sinn, wenn wir uns erst einmal über den Kritikbegriff verständigen - allerdings bin ich mir nicht sicher, ob wir eine explizite gesellschaftstheoretische Basis voraussetzen sollten. Teilweise waren ja Forderungen zu der Zeit, als sie geäußert wurden, vielleicht kritisch und radikal, erscheinen aber aus heutiger Perspektive nicht mehr so (ohne jetzt von einem wissenschaftlichen Fortschrittsideal ausgehen zu wollen). Für den Städtebau gilt natürlich weitgehend die Idee, das Chaos der Städte zu ordnen und planerisch oder baulich zu besseren Lebensverhältnissen beizutragen - also letztlich ein sozialreformerischer, angewandter Ansatz. Darüber hinaus gab und gibt es ja noch eine Reihe von idealen Architekturund Stadtmodellen, die als Hegemoniekritik und radikale Neuordnungen verstanden werden können (manche Modelle befassen sich ja durchaus mit Besitzverhältnissen o. Ä.).

Hier ist vielleicht auch noch interessant zu erwähnen, dass es in der Architektur auch immer einen ästhetisch-gestalterisch geprägten Kritikbegriff gibt. Zum Beispiel gab es in den 1980er Jahren eine Debatte zur kritischen Architektur, die von Peter Eisenman geprägt wurde. Als kritische Gestaltung galt, was sich nicht durch den Status Quo (also durch den Kapitalismus) vereinnahmen ließ. Rem Koolhaas zog diese Möglichkeit in Zweifel: Architektur könne per se nicht kritisch sein, die Möglichkeit der Vereinnahmung bestehe immer.

Letztendlich bleibt natürlich die Frage, ob ein formaler beziehungsweise ästhetischer Ansatz in der Architektur - also das Objekt selbst - kritisch sein kann, oder ob es produktiver ist, über soziale, politische und ökonomische Veränderungen nachzudenken. Mir scheint Letzteres sinnvoller - ohne dass ich mich dabei von Ästhetik und Formalem grundsätzlich abwenden möchte. Meines Erachtens geht es aber viel mehr um eine Reflexion kritischer Praxis in der Architektur und Stadtplanung, die sich ja nie im luftleeren Raum abspielt. Die Frage scheint mir zentral, welche Wechselwirkungen es gibt zwischen sozialen Kontexten, die von Macht, Ausbeutung, Ungleichheit oder Ähnlichem geprägt sind, auf der einen Seite, und dem Planen und Bauen auf der anderen. 
$\boldsymbol{S H}$ : Schaut man in der deutschsprachigen kulturhistorischen Urbanisierungsforschung nach sich als kritisch ausweisenden Ansätzen und Perspektiven, so wird man kaum fündig werden. Ich habe lange recherchiert und letztlich auch einige Professor_innen der Stadtgeschichte gefragt, ob sie Ansätze und Perspektiven kennen, die sich selbst als kritisch verstehen, und zwar nicht nur in einem erkenntnistheoretischen, sondern auch in einem gesellschaftsanalytischen Sinne. Dies scheint jedoch wirklich nicht der Fall zu sein. Dies ist selbst für eine gemeinhin als eher konservativ geltende Disziplin wie der Geschichtswissenschaft bemerkenswert, wo es doch beispielsweise im Umfeld der Zeitschrift WerkstattGeschichte eine rege Diskussion um kritische Ansätze gab. Ebenso gibt es doch in den Feldern feministischer oder post-marxistischer Geschichtswissenschaften Ansätze, die sich selbst als ,kritisch 'bezeichnen und durchaus auch städtische Phänomene erforschen. Warum dies in der deutschen wie auch anglo-amerikanischen Stadtgeschichte nur sehr eingeschränkt der Fall ist, darüber kann ich nur spekulieren. So wäre zu vermuten, dass sich diese Studien strategisch eher im Feld der Sozialgeschichte verorten, wo es auch stärkere institutionelle Strukturen gibt als in der Stadtgeschichte, der zudem oftmals noch ein eher provinzieller Ruf anhaftet. Allerdings finden sich auch im Feld der historischen Urbanisierungsforschung immer wieder herrschaftskritische Studien zu Kolonialismus oder Analysen zur Klassendynamik urbaner Transformationen et cetera. Ebenso entsteht vor allem ab den 1990ern eine Reihe von produktiven Arbeiten, die sich beispielsweise aus Gender-Perspektive der historischen Stadtforschung nähern oder durch Foucault und Bourdieu inspiriert sind.

Zudem hat sich unter dem Eindruck der kulturgeschichtlichen Wende wie des spatial turns in den letzten Jahren die historische Urbanisierungsforschung stark ausdifferenziert und bietet eine Vielzahl neuer produktiver Ansätze. So finden sich mittlerweile Studien zur urbanen Umweltgeschichte ebenso wie (post-)koloniale Studien, Arbeiten zur Rolle von Kriegen und Katastrophen für die Urbanisierung, Demographie und Gesundheit, zur Geschichte urbaner Repräsentationsformen und Bildpolitiken, local Governance und Selbstverwaltung, Stadt- und Infrastrukturen und vieles mehr. Zugleich wird auch der stark eurozentrische Blick der bisherigen Forschungen immer deutlicher erkannt und das führt vermehrt dazu, dass sich nun beispielsweise auch der osteuropäischen oder asiatischen Urbanisierungsgeschichte zugewandt wird. Gerade die in den letzten Jahren verstärkt unternommenen Bestrebungen, die ,globalen'Verbindungen von historischen Urbanisierungsbewegungen herauszuarbeiten, erscheinen mir als wichtige Korrektive gegenüber der oft noch sehr im Lokalistischen und Provinziellen verhafteten klassischen Stadtgeschichte. Diese Ansätze erlauben es nicht zuletzt, den konzeptuellen wie methodologischen Nationalismus wie den Regionalismus der Stadtgeschichtsforschung zu dekonstruieren und neue Perspektiven zu eröffnen.

Da sich auch die historische Urbanisierungsforschung zudem in enger Wechselwirkung mit gesellschaftlichen Transformationen befindet, ließe sich angesichts der zunehmenden Bedeutung urbaner Fragestellungen auch eine Renaissance der historischen Stadtforschung erwarten, die auch Raum für herrschaftskritische und emanzipatorische Forschungen bietet. 
$\boldsymbol{B M}$ : Wenn ich das richtig sehe, dann ist kritische Stadtgeographie und zumal kritische deutschsprachige Stadtgeographie etwas, das recht wenig Geschichte hat. Das, was es an Geschichte gibt, ist weder kritisch noch deutschsprachig noch stadtgeographisch. Ich glaube nicht, dass der Begriff vor der Mitte der 1990er Jahre irgendwo in einer deutschsprachigen Publikation aufgetaucht ist. Aber das mag vielleicht auch damit zu tun haben, dass diejenigen, die so etwas betrieben haben, ihr Tun nicht unbedingt disziplinär so eng gefasst haben und vielleicht auch gar nicht ,Stadt' als so zentral begriffen haben. Aber wenn man sich anschaut, wie ,Stadt ' und ,kritisch - sowohl als Begriffe wie auch als eine irgendwie in Tradition einer kritischen Gesellschaftstheorie stehende Perspektive - in die Geographie kamen, dann gibt es da doch eine Reihe interessanter Beobachtungen.

Die alte Stadtgeographie und die geographische Auseinandersetzung mit Stadt waren, wie ich vorhin beschrieben habe, alles andere als kritisch - außer vielleicht ,stadtkritisch'. Und wenn für die Soziologie die Stadt sicherlich zentral für die sie interessierenden Formen der Vergesellschaftung war, so waren Geograph_innen auch nach 1945 eher an gemeinschaftlichen Formen, an Dörfern, interessiert. Das ist nicht überraschend, wurde doch Geographie vor 1945 nicht und auch danach zunächst nur schleppend als Sozialwissenschaft gedacht.

Das war aber sicherlich die Grundbedingung für so etwas wie eine Kritische Geographie. Versuche einer solchen kritischen und gesellschaftswissenschaftlichen Geographie, die parallel zu einer stärker angewandten, planerischen und quantitativ-theoretischen Geographie in den späten 1960er Jahren entstanden, wurden aber durch die dominanten Positionen in der Disziplin weitgehend isoliert. Während sich eine angewandte und szientistische Geographie ab den 1970er Jahren als Modernisierung des Faches langsam durchsetzen konnte, wurde eine gesellschaftskritische Perspektive weitgehend verhindert und verdrängt. Dort, wo eine Kritische Geographie stattfand, war sie eher mit allgemeiner Wissenschaftstheorie beschäftigt und vielleicht auch mit Gesellschaftstheorie als mit empirischer oder theoretischer Stadtforschung.

Natürlich gab es die eine oder andere Publikation, etwa die Arbeiten von H. D. von Frieling und eine Reihe von Beiträgen in der Reihe „Urbs et Regio“ oder den Sammelband Theorien zur Stadtentwicklung von Hartmann, Hitz, Schmid und Wolff in den frühen $1980 e r$ Jahren. Auch in den meist sehr kurzlebigen linken Zeitschriften wie Roter Globus, Geografiker oder Geographie in Ausbildung und Planung, die in den 1970er Jahren erschienen, gab es gelegentlich das, was man als kritische Stadtgeographie bezeichnen könnte. Etwa Beiträge mit Titeln wie „Das Ghetto als interne Neokolonie“, eine Übersetzung aus der Antipode, oder Texte zu städtischen Problemen in der ,Dritten Welt'. Kritisch heißt hier ziemlich ausschließlich: marxistisch. Die ersten feministischen Beiträge erscheinen in den späten 1980er Jahren, aber die begriffen sich sicherlich kaum als eine kritische ,Stadt'-geographie.

Kritische Stadtgeographie entstand anderswo. Vermutlich kann man ohne großes Risiko David Harveys Social Justice and the City von 1973 als den Gründungstext für das bezeichnen, was heute unter dem Label Kritische Stadtgeographie läuft. Bis das in die deutschsprachige Geographie kam, vergingen noch einige Jahrzehnte, und von vielen der in den 1970er 
Jahren sozialisierten Geograph_innen wurden die 1980er Jahre als eine ziemlich bleierne Zeit beschrieben. Und selbst hier ist nicht so ganz klar, ob die Impulse wirklich aus der deutschsprachigen Geographie kamen. Erste Übersetzungen von Harveys stadtgeographischen Arbeiten erschienen eher bei Planer_innen (Stadtbauwelt 1974), Soziolog_innen wie Krämer und Neef oder in einer Zeitschrift wie der prokla (1987). In deutschsprachigen geographischen Zeitschriften wurde das fast nicht rezipiert.

Eine Traditionslinie kritischer Stadtgeographie reicht vermutlich nicht in die Zeit vor Harvey zurück. Ich würde stark vermuten, dass, wenn kritische Stadtgeograph_innen sich heute auf ältere Texte - also sagen wir mal auf jene aus einer Zeit von vor Harvey und Lefebvre - beziehen, dies eher die Texte von Autoren wie Marx, Engels, Simmel oder Benjamin sind.

NG: Die erste Übersetzung von Harvey ins Deutsche erschien meines Wissens als Beiheft der zweiten Auflage der Publikation Sanierung für Wen? des Büros für Stadtsanierung und soziale Arbeit. Rolf Czeskleba-Dupont, ein Soziologe von der FU Berlin, der im Büro für Stadtsanierung eng mit Architekt_innen zusammenarbeitete, übersetzte 1972 Harveys Revolutionäre und gegenrevolutionäre Theorie in der Geographie und die Probleme der Ghettobildung. Und Castells wurde ja, wie vorhin schon erwähnt, erstaunlicherweise zunächst von Architekt_innen übersetzt.

$\mathbf{s} \backslash \mathbf{u}:$ Es scheint ja so, als wären die späten 196oer Jahre in allen Erzählungen recht entscheidend gewesen. Ist das, was wir heute,kritische Stadtforschung nennen würden, erst in den 196oern entstanden? Und bezeichnen wir damit heute dieselbe Forschung, die sich schon damals als, kritisch'verstanden hat? Dies bringt ja auch die Frage nach den Produktionsbedingungen kritischer Wissenschaft ins Spiel. Bei aller Institutionenkritik waren die Universität ebenso wie Netzwerke doch sicherlich wichtige Orte für kritische Stadtforschung. Wie würdet ihr denn die Institutionalisierung einer (kritischen) Stadtforschung in euren Disziplinen beschreiben?

BM: Als Initial einer Kritischen Geographie gilt gemeinhin ein Zusammenschluss von Studierenden, die die Zeitschrift Geografiker herausgegeben und auf dem Geographentag 1969 in Kiel einigen Ärger gemacht haben. Der Kieler Geographentag 1969 ist so ein mythisches Ereignis in der Geographie. Aber wie gesagt, deren Fokus war eher allgemeingeographisch. In den 1980er Jahren gab es Netzwerke wie WISSKRI, eine Gruppe kritischer Geograph_innen, und auch ein erstes Netzwerk feministischer Geograph_innen hat sich in dieser Zeit gegründet. Stadt blieb aber auch da eher ein Randthema. Themen wieÖkologie und ,Dritte Welt‘ waren sicherlich für die Geographie die bestimmenderen.

NiS: Ich würde sagen, dass eine Stadtsoziologie, die sich aufgrund ihrer Forschungsausrichtung explizit als kritisch verstanden hat, von den späten 1960er Jahren bis in die 1980er Jahre hinein ihre Blütezeit hatte und sich danach wieder allzu stark in sozialreformerische Politiken hat einbinden lassen. Ich denke dabei vor allem an die erfolgreiche Anregung und Begleitung des Programms „Soziale Stadt“ in den 1990ern, das ohne eine stadtsoziologische Segregationsforschung und quartiersbezogene Partizipationsansätze, die auch 
innerhalb der neuen Planungsfakultäten entstanden, kaum denkbar gewesen wäre. Dennoch wurden immer wieder auch explizit gesellschaftskritische Arbeiten verfasst, wenn sie auch nie in der Mehrheit waren. Dazu gehören auch die zunächst kämpferisch polarisierenden feministischen Stadtkritiken. Dieser Bereich hat besonders wenig Institutionalisierung erfahren (beispielsweise im Rahmen fester Stellen oder Denominationen von Professuren). Feministische Lehr- und Forschungsinhalte fanden sich an Universitäten allenfalls, weil einzelne Wissenschaftlerinnen zusätzlich zu den üblichen Themen entsprechende Forschungsschwerpunkte hatten. Allerdings haben sich an vielen Hochschulen in den 1990er Jahren Zentren für Gender Studies gegründet, an deren thematischer Konturierung auch Stadt- und Raumsoziologinnen maßgeblich beteiligt waren, zum Beispiel in Frankfurt am Main, Kassel und Marburg.

Das Verschwinden der meisten Professuren für Stadtsoziologie in grundständigen Soziologiestudiengängen seit den 1990er Jahren beantwortet die Frage nach dem institutionellen Ort für (kritisches) stadtsoziologisches Wissen eindeutig: Stadtsoziologie ist insgesamt an deutschsprachigen Unis derzeit vor allem Hilfswissenschaft oder ,Grundlagenfach in Planungsstudiengängen, in denen die Studierenden auf einen ,Anwendungsbezug' pochen und dabei kaum Interesse an kritisch-theoretischen Auseinandersetzungen mit dem Bestehenden haben.

NG: An den Architekturfakultäten hat sich im Zuge der großen Umstrukturierungen als Folge der Studentenbewegung in den 1968ern ein institutioneller Wandel vollzogen (Fakultäten wurden in Fachbereiche aufgespalten, deren Titel sich teils gar nicht mehr auf ,Architektur' bezogen, sondern Namen trugen wie ,Bauplanung' und ,-fertigung'). In dieser Zeit vollzog sich ja auch an einigen Universitäten die Abspaltung der Stadt- und Regionalplanungs-Fachbereiche von der Architektur. Seither ist das Fach ,Städtebau' sowohl an Architektur- als auch an Planungsfakultäten verankert. In der Architektur selbst wurden neue Fachgebiete und Ansätze in die Lehre integriert (mehr Theorie, weitere Grundlagenfächer). Heute werden an einigen Universitäten die Theorielehrstühle wieder etwas zusammen gestrichen. Was ich neben der Institutionalisierung für wichtig halte, sind eine Reihe von kritischen Netzwerken, vor allem in den 1960/70er Jahren, die teils marxistisch oder anarchistisch geprägt waren, wie die Rote Zelle Bau oder die Marxistisch-Leninistische-Hochschulgruppe Bau. Zu dieser Zeit gab es auch eine Gruppe, die sich in der Gewerkschaft „Bau Steine Erden“ für die Architekt_innen als ,lohnabhängige Arbeiter" engagierte. Es wurden Kollektive oder Gesellschaften gegründet, die die Büroarbeit anders organisierten. Ich vermute, dass es in den 1980/90er Jahren auch noch Gruppen gab, von denen ich allerdings nichts weiß. Für die frühen 2000er ist das Architekturkollektiv „Freies Fach“ zu nennen, das dann auch die kritische Architekturzeitschrift AnArchitektur herausgab.

SH: Die Situation der Stadtgeschichte ist dem nicht unähnlich. Auch hier sind die Professuren mittlerweile wieder sehr rar gesät. Sich dezidiert als kritisch verstehende Forschung ist dabei bestenfalls am Rande auszumachen. Allerdings sind durch die zunehmende Internationalisierung der Forschung beispielsweise durch die European Association for Urban History seit den 
1990er Jahren sowie auch die Gründung der Gesellschaft für Stadtgeschichte und Urbanisierungsforschung (GSU) neue institutionelle Zusammenhänge entstanden, die durchaus Räume, Ressourcen und Netzwerke für solche Forschungen und Kollaborationen freigeben könnten. Diese gilt es zu nutzen.

$\boldsymbol{B M}$ : Vermutlich ist das in der Geographie ein wenig anders. Es ist nicht so, dass Stadtgeographie einen Niedergang erlebt hätte und ich glaube nicht, dass sich die Zahl der stadtgeographischen Professuren in den vergangenen Jahren reduziert hätte. Und wenn man sich anschaut, wie sich Geograph_innen heute selbst positionieren, so ist doch die Beschreibung der eigenen Arbeit als Stadtgeographie ziemlich häufig zu finden, und das gilt sicherlich ganz besonders für Leute, die sich in der Tradition einer Kritischen Theorie der Gesellschaft verorten würden.

$\mathbf{s} \backslash \mathbf{u}:$ Bisher haben wir eine recht akademische Geschichte gehört. Bei $\mathrm{s} \mathrm{u} \mathbf{b} \backslash \mathbf{u} \mathbf{r} \mathbf{b}$ a $\mathbf{n}$ versuchen wirja immer wieder - auch wenn das nicht immer klappt-, eine Beziehung zwischen Akademie und Aktivismus herzustellen (ohne da jetzt so eine klare Trennlinie aufstellen zu wollen). Fällt euch dazu etwas in der Geschichte ein? Gingen die kritischen Stadtforscher_innen an die Fließbänder und vor die Fabriktore?

NG: Einige Architekt_innen und Städtbauer_innen waren auf jeden Fall auch Aktivist_innen. Manches, was ich weiter oben beschrieben habe, wie zum Beispiel die Bewegung diverser Basisgruppen in die Stadtteilarbeit, deutet ja schon darauf hin. Viele Zeitzeug_innen haben uns im Rahmen des Buchs Vergessene Schulen von regelmäßigen Demonstrationen, dem Verteilen von Flugblättern - teilweise direkt vor den Werkstoren großer Firmen - und anderen Aktionen erzählt. Eine Person hat an der Seite von Rebellen im Bürgerkrieg von Angola gekämpft und von anderen haben wir gehört, dass sie zumindest für eine Zeit lang an die Fließbänder gingen. Ansonsten gab es in der Architektur in den 1960ern und 1970ern auch eine intensive Auseinandersetzung mit den Arbeitsverhältnissen von Architekten als lohnabhängigen Arbeitern, wie ich das schon oben erwähnt habe. Parallel dazu haben sich einige sehr stark in der Gewerkschaft „Bau Steine Erden" engagiert oder andere kollektiv oder genossenschaftlich arbeitende Bürogemeinschaften gegründet. Der Anspruch, die gesellschaftlichen sowie die eigenen beruflichen Praktiken zu verändern, war damals weit verbreitet. Letztlich sollten so aber auch die akademischen Praktiken verändert werden. Es gab verschiedene selbstorganisierte Lehr- und Lernformate, die auch zu einem Wandel an den Architekturfakultäten beitragen sollten.

NiS: Ich gehe davon aus, dass viele Soziolog_innen in die neuen Projekte gingen: die linken und die feministischen Wohnprojekte, Hausbesetzungen, Wohn- und Arbeitskollektive und -genossenschaften, die seit den 1960er Jahren entstanden sind und teilweise bis heute existieren. In dieser Praxis des Sich-Auskoppeln-Wollens und Neues-Gestalten-Wollens finden sich deutliche Verbindungen zu kritischen, akademischen Auseinandersetzungen, was eben sicherlich auch daran liegt, dass viele der Akteur_innen studiert haben oder sich zumindest in ihrer Freizeit in Lesekreisen mit viel Theorie beschäftigt 
haben. Und auch in der Stadtteilarbeit waren ja sicher viele von ihnen engagiert, auch zusammen mit Raumplaner_innen und Sozialpädagog_innen. Genaueres weiß ich dazu allerdings nicht, und das ist bislang, soweit ich weiß, auch nicht systematisch erforscht worden. Eure Zeitzeugenforschung mit den wilden Architekt_innen der 1968er Jahre ist da sicher bahnbrechend.

$\boldsymbol{S H}$ : Es gab in den lokalen Geschichtswerkstätten der 1970er und 1980er eine Vielzahl von Gruppen, die eng mit sozialen Bewegungen zusammengearbeitet und sich Themen wie städtischen Kämpfen, Wohnungsnot etc. gewidmet haben. Hier ging es also um eine Art ,Stadtgeschichte von unten“. Auch heute gibt es in vielen Städten Gruppen wie Berlin Postkolonial e.V., die sich kritisch mit der urbanen Kolonialgeschichte auseinandersetzen. Diese organisieren nicht nur Stadtführungen, Veranstaltungen und erinnerungspolitische Initiativen, sondern machen auch Forschungsarbeit und geben Bücher heraus. Diese Forschungen finden aber bezeichnenderweise bislang weitestgehend außerhalb der Universitäten statt und werden innerhalb der Institutionen noch viel zu wenig rezipiert.

$\boldsymbol{B M}$ : Ich weiß es nicht. Kritische Geograph_innen haben sowas sicherlich gemacht und natürlich auch an städtischen sozialen Bewegungen und Konflikten teilgenommen. Aber dass das Teil des kollektiven Gedächtnisses geworden ist, glaube ich nicht. Ich glaube, es gibt doch noch so einiges an Ausgrabungsarbeit zur Geschichte der Kritischen Stadtforschung und der Kritischen Geographie insbesondere zu tun.

$\mathbf{s} \backslash \mathbf{u}:$ Was wünscht ihr euch für eure jeweilige Disziplin, um kritische Stadtforschung zu stärken? Und was fehlt? Andere Arbeitsbedingungen, andere Forschungsfoki, andere Förderungsmöglichkeiten?

NiS: Für eine kritische soziologische Stadtforschung reicht es nicht, beispielsweise Armut und soziale Ungleichheit in den Städten zu benennen - mehr Forschung zu ihrer Reproduktion und Ausweitung wäre allerdings schon mal etwas. Auch die Forderung nach städtischer ,sozialer Mischung' und ,Integration ' bezeugt häufig das Fehlen einer herrschaftskritischen Auseinandersetzung und entsprechender gesellschaftstheoretischer Fundierung. Eine Reflexion zu Machtverhältnissen und Hegemonien, aber auch zu Demokratiedefiziten und problematischen städtischen Entwicklungen, etwa im Bereich der ,Sicherheitspolitik ${ }^{\natural}$, brächte eine radikalere Forderung oder Haltung gegenüber den herrschenden (immer stärker und deutlicher ökonomisch grundierten) Verhältnissen mit sich, und damit ein entschlosseneres Eintreten gegen soziale Ungleichheiten in vielen gesellschaftlichen Kontexten, auch in den Städten. Die vertretenen soziologischen Positionen sind erstaunlich blass und fast immer einseitig bürgerlich, was eigentlich nicht reflektiert wird. Dabei fehlen in stadtsoziologischen Arbeiten marginalisierte Perspektiven - die von Arbeiter_innen, Zugewanderten, People of Colour, Frauen, Queers, Menschen mit Behinderung, Kapitalismusgegner_innen.

$\boldsymbol{S H}$ : Diese Forderungen kann ich auch für die historische Urbanisierungsforschung uneingeschränkt unterschrieben. Auch hier ginge es darum, 
herrschaftskritische und gesellschaftstheoretische Ansätze zu stärken wie auch marginalisierte Perspektiven jenseits der immer noch erstaunlich dominanten bürgerlichen Erzählung zu befördern. Wenn es stimmt, dass die Fragen und Themen der Geschichtswissenschaft aus den jeweils gegenwärtigen Problemen und Debatten heraus motiviert sind, so muss dies auch für die historische Stadtforschung gelten. Demzufolge wären beispielsweise die Perspektivierung auf globale Migrationsdynamiken und eine De-Zentrierung europäischer Stadtgeschichte ebenso wichtig wie Untersuchungen zur Geschichte städtischer Regierungstechniken und urbaner Subjektivierungsweisen, wie sie mittlerweile verstärkt in der angloamerikanischen Forschung unternommen werden. Stattdessen scheint sich zumindest nach meiner Einschätzung der Trend zu einer unkritischen deutschsprachigen Stadtgeschichte auch bei jüngeren Forscher_innen bis auf wenige Ausnahmen weiter fortzusetzen. Dies zeigt sich nicht zuletzt in einer starken Fokussierung auf Akteursgeschichten sowie einer verkürzt kulturgeschichtlichen Perspektive, die ein bemerkenswertes Desinteresse an Fragen der politischen Ökonomie oder historischen Herrschafts- und Ausbeutungsverhältnissen hat.

Zudem erscheint mir die historische Urbanisierungsforschung auch sehr dafür geeignet, die Reichweite von dominanten Modellen und Konzepten der Stadt- und Raumforschung auszuloten. Hier wäre beispielsweise zu fragen, welche Erklärungskraft den Theorien Lefebvres, Castells und anderen eigentlich in nicht-kapitalistischen Kontexten zukommt und welche Modelle hier vielleicht brauchbarer wären.

$\boldsymbol{B M}$ : Ich bin vielleicht etwas optimistischer. Mein Eindruck ist der, dass in der deutschsprachigen Geographie eine kritische Perspektive auf Stadt und Urbanität heute stärker vertreten ist als je zuvor. Oft wird in der Geographie dabei die These vertreten, dass es paradoxerweise die Forderung war, sich im Zuge der neoliberalen Umstrukturierung zu internationalisieren. Da die anglophone Geographie aber recht stark von kritischen Autor_innen geprägt wurde und wird, war es auf einmal möglich, mit marxistischen und feministischen Positionen am Exzellenzspiel teilzunehmen. Wie weit diese Geschichte trägt, ist mir nicht ganz klar, aber was vielleicht fehlt, in der disziplinären Praxis, aber auch in unserem Gespräch, ist die Frage der Vermittlung und der Lehre. Was heißt ,Kritische Stadtforschung' in der ,kritischen“ universitären Lehre? Wie Thomas Bürk in dem Gespräch mit uns (in diesem Heft) ja so schön sagt, kann es nicht nur darum gehen, Harvey zu lesen.

NG: In der Architektur und im Städtebau heute fehlen auch weitgehend die marginalisierten Perspektiven, die Nina S. gerade angesprochen hat. Ansonsten kann ich gerade ein eher gemischtes Bild beobachten. Bestimmte Themen wie Wohnungsnot, soziale Bewegungen, städtische Konflikte, Migration und Möglichkeiten einer alternativen, das heißt nicht marktkonformen, Stadtentwicklung werden in der Architektur und im Städtebau gerade wieder etwas verstärkt diskutiert. Das finde ich grundsätzlich positiv und es scheint mir, dass dadurch vielleicht sogar etwas Raum für eine kritische Praxis gewonnen werden kann. Gleichzeitig erscheinen mir einige Debatten, zum Beispiel um ,soziale Architektur‘ oder die ,selbstgemachte Stadt‘ etwas 
verkürzt. Hier würde ich mir wünschen, dass Produktionsbedingungen, Machtverhältnisse und Ausschlussprinzipien etwas weiter mitgedacht würden (siehe auch meinen Beitrag zusammen mit Hannes Langguth und Mario Schulze in s u b $\backslash \mathbf{u} \mathbf{r} \mathbf{b}$ a $\mathbf{n}$ Heft 3/3).

$\mathbf{s} \backslash \mathbf{u}$ : Vielen Dank für diese interessante Diskussion, die einen Einblick in die Geschichte der deutschsprachigen (kritischen) Stadtforschung und die Bedingungen ihrer Produktion gibt. Ich glaube, dass eure Überlegungen schon eine gute Grundlage für eine Diskussion zu den Produktionsbedingungen der Stadtforschung und zu ihrer Interdisziplinarität darstellen. Eure historische Rekonstruktion könnte sicherlich präzisiert, vertieft und erweitertwerden. Man könnte zum Beispiel fragen, ob die Relevanz der Kontakte zwischen der deutschsprachigen Stadtforschung und der globalen Zirkulation von Ideen und wissenschaftlichen Praktiken, die ihr besonders stark in der zweiten Hälfte des 20. Jahrhunderts verortet habt, nicht vordatiert werden müsste.

Die Frage der Interdisziplinarität betreffend setzt eure gesamte Herangehensweise überhaupt die Existenz von Disziplinen voraus. Man könnte aber auch fragen, ob es überhaupt Sinn macht, eine solche Analyse aus disziplinären Perspektiven zu betreiben, wenn viele der Autor_innen, die genannt worden sind, sich schon immer an den Grenzen der Disziplinen bewegt haben. Kurz gefasst könnte man fragen, ob das Problem der Multidisziplinarität und daher das Bedürfnis nach Interdisziplinarität nicht eher eine sehr junge historische Entwicklung ist.

In eurer Rekonstruktion der Geschichte der deutschsprachigen Stadtforschung habt ihr aufeinige weiße Flecken hingewiesen, darunter scheint eine tiefere Auseinandersetzung mit der Stadtforschung der DDR für euch alle ein Desiderat zu sein. Ein weiterer Punkt, der in der Diskussion offen blieb, ist die Definition von Kritik und von, kritisch'. In dieser Hinsicht würde es sich zu fragen lohnen, ob man einen absoluten Begriff von Kritik braucht, der a priori benutzt werden kann, oder ob man eher einen, situativen'Begriff von Kritik, dervon der Selbstverortung der Wissenschaftler_innen ausgeht, anwenden sollte. Um diese Frage zu beantworten, sollte man auch den strategischen Nutzen der Bezeichnung ,kritisch'hinterfragen und die Rolle, die auch $\mathrm{s} \mathbf{u} \mathbf{b} \backslash \mathbf{u} \mathbf{r} \mathbf{b}$ a $\mathbf{n}$ in diesem Kontext für sich beansprucht.

Wir hoffen, mit diesen und weiteren offenen Punkten, die sich aus dieser ersten Diskussion zur kritischen Stadtforschung ergaben, eine Debatte zu starten, die in den kommenden Monaten eine Fortsetzung findet.

\section{Beteiligte}

Nina Gribat ist Stadt- und Planungsforscherin. Sie arbeitet zur Zeit an international vergleichenden Forschungsprojekten, die sich mit Stadtentwicklungskonflikten, schrumpfenden Städten und den Studienreformen/-revolten um 1968 in der Architektur beschäftigen.

Stefan Höhne ist Historiker und Kulturwissenschaftler. Er forscht und lehrt zu transatlantischer Stadtgeschichte, Kulturgeschichte der Technik und Infrastruktur sowie seit Neuerem zu Praktiken der Sabotage im Kalten Krieg. stefan.hoehne@metropolitanstudies.de 
Boris Michel ist Geograph. Er forscht zur Geschichte der Geographie und interessiert sich dabei zunehmend für die Geschichte der geographischen Auseinandersetzung mit Stadt. boris.michel@fau.de

Nina Schuster ist Soziologin und forscht an der Schnittstelle von stadtsoziologischen und queer/feministischen Theorien zur sozialen und räumlich-materiellen Produktion sozialer Ungleichheit.

nina.schuster@tu-dortmund.de 
